Pacific

Journal of

Mathematics

CONNECTED SUMS OF CLOSED RIEMANNIAN MANIFOLDS AND FOURTH-ORDER CONFORMAL INVARIANTS

DAVID RASKE 


\title{
CONNECTED SUMS OF CLOSED RIEMANNIAN MANIFOLDS AND FOURTH-ORDER CONFORMAL INVARIANTS
}

\author{
DAVID RASKE
}

In this note we take some initial steps in the investigation of a fourth-order analogue of the Yamabe problem in conformal geometry. The Paneitz constants and the Paneitz invariants considered are believed to be very helpful to understand the topology of the underlying manifolds. We calculate how those quantities change, analogous to how the Yamabe constants and the Yamabe invariants do, under the connected sum operations.

\section{Introduction}

Let $(M, g)$ be a connected compact Riemannian manifold without boundary of dimension $n \geq 5$. Let

$$
Q[g]=-\frac{n-4}{4(n-1)} \Delta R+\frac{(n-4)\left(n^{3}-4 n^{2}+16 n-16\right)}{16(n-1)^{2}(n-2)^{2}} R^{2}-\frac{2(n-4)}{(n-2)^{2}}|\mathrm{Ric}|^{2}
$$

be the so-called $Q$-curvature, where $R$ is the scalar curvature, Ric is the Ricci curvature. And let

$$
P[g]=(-\Delta)^{2}-\operatorname{div}_{g}\left(\left(\frac{(n-2)^{2}+4}{2(n-1)(n-2)} R g-\frac{4}{n-2} \operatorname{Ric}_{g}\right) d\right)+Q[g]
$$

be the so-called the Paneitz-Branson operator. It is known that

$$
P[g] u=Q\left[g_{u}\right] u^{\frac{n+4}{n-4}}
$$

which is called the Paneitz-Branson equation, where $g_{u}=u^{\frac{4}{n-4}} g$ (see [Paneitz 1983; Branson 1987; Xu and Yang 2001; Djadli et al. 2000]). We consider the equation (1-3) as a fourth-order analogue of the well-known scalar curvature equation

$$
L[g] v=R\left[g_{v}\right] v^{\frac{n+2}{n-2}},
$$

where

$$
L[g]=-\frac{4(n-1)}{n-2} \Delta+R
$$

MSC2010: primary 57R65; secondary 57R99.

Keywords: Paneitz-Branson operator, $Q$-curvature. 
is the so-called conformal Laplacian and $g_{v}=v^{\frac{4}{n-2}} g$. The well-known Yamabe problem in conformal geometry is to find a metric, in a given class of conformal metrics, which is of constant scalar curvature, i.e., to solve

$$
L[g] v=Y v^{\frac{n+2}{n-2}}
$$

on a given manifold $(M, g)$ for some positive function $v$ and a constant $Y$. The affirmative resolution to the Yamabe problem was given in [Schoen 1984] after other notable works [Yamabe 1960; Trudinger 1968; Aubin 1976]. In fact, it was proven that there exists a so-called Yamabe metric $g_{v}$ in the class $[g]$ which is a minimizer for the so-called Yamabe functional

$$
Y(v)=\frac{\int_{M}(v L[g] v) d v_{g}}{\left(\int_{M} v^{\frac{2 n}{n-2}} d v_{g}\right)^{\frac{n-2}{n}}} .
$$

In this paper we investigate a fourth-order analogue of the Yamabe problem. Let $C_{+}{ }^{\infty}(M)$ be the space of smooth positive functions on $M$. Similar to the Yamabe problem, we define the Paneitz functional

$$
\wp_{g}(u)=\frac{\int_{M}(u P[g] u) d v_{g}}{\left(\int_{M} u^{\frac{2 n}{n-4}} d v_{g}\right)^{\frac{n-4}{n}}}
$$

for $u \in C_{+}^{\infty}(M)$ and the Paneitz constant associated with $(M,[g])$

$$
\lambda(M,[g])=\inf _{u \in C_{+}^{\infty}(M)} \wp g(u) .
$$

It is clear that $\lambda(M,[g])$ is a conformal invariant of the conformal class $[g]$ because of the conformally covariant property of the Paneitz-Branson operator:

$$
P\left[g_{w}\right] u=w^{-\frac{n+4}{n-4}} P[g](w \cdot u)
$$

where $g_{w}=w^{\frac{4}{n-4}} g \in[g]$. To describe the differential structure of $M$, we define

$$
\lambda(M)=\sup \lambda(M,[g]) .
$$

We will refer to $\lambda(M)$ as the Paneitz invariant of the manifold $M$ as the counterpart of Yamabe invariant. In [1986], Gil-Medrano studied the Yamabe constant for a connected sum of two closed manifolds. One interesting consequence of connected sum results in [Gil-Medrano 1986] is that every compact manifold without boundary admits a conformal class of metrics whose Yamabe constant is very negative. In Section 3 we calculate as Gil-Medrano did in [1986] to verify that

Theorem 1.1. Let $\left(M_{1}, g_{1}\right)$ and $\left(M_{2}, g_{2}\right)$ be two compact Riemannian manifolds of dimension $n \geq 5$. Then, for each $\epsilon>0$, there is a conformal class $[g]$ of metrics 
on $M_{1} \# M_{2}$ such that

$$
\lambda\left(M_{1} \# M_{2},[g]\right)<\min \left\{\lambda\left(M_{1},\left[g_{1}\right]\right), \lambda\left(M_{2},\left[g_{2}\right]\right)\right\}+\epsilon
$$

and there exists a conformal class $[h]$ of metrics on $M_{1} \# M_{2}$ such that

$$
\lambda\left(M_{1} \# M_{2},[h]\right)<2^{-\frac{n-4}{n}}\left(\lambda\left(M_{1},\left[g_{1}\right]\right)+\lambda\left(M_{2},\left[g_{2}\right]\right)\right)+\epsilon .
$$

Due to the works of Schoen and Yau [1979] (see also [Gromov and Lawson 1980]), one knows that there is some topological constraint for a manifold to possess a metric of positive Yamabe constant. Therefore it is interesting to see how the Yamabe invariant is effected by connected sum. It was proven in [Kobayashi 1987], [Schoen and Yau 1979], and [Gromov and Lawson 1980] that the Yamabe invariant of connected sum of two manifolds with positive Yamabe invariants is still positive. More precisely, Kobayashi in [1987] showed that the Yamabe invariant of connected sum of two manifolds is greater than or equal to the smaller of the Yamabe invariants of the two. In Section 4 we obtain an analogue for the Paneitz invariant.

Theorem 1.2. If $M_{1}$ and $M_{2}$ are compact manifolds of dimension $n \geq 5$, then

$$
\lambda\left(M_{1} \# M_{2}\right) \geq \min \left\{\lambda\left(M_{1}\right), \lambda\left(M_{2}\right)\right\} .
$$

The positivity of Paneitz invariant in dimension higher than 4 should be a topological constraint, as indicated by successful researches in [Chang and Yang 2002] (references therein) for a fourth-order analogue of how Gaussian curvature influences the geometry of surfaces in dimension 2. Another testing ground is to consider closed locally conformally flat manifolds. Then the recent works in [Chang et al. 2004] and [González 2005] indicate to us that the positivity of fourth-order curvature is indeed very informative about the topology of the underlying manifolds. We would also like to mention the work by $\mathrm{Xu}$ and Yang in [2001] where they demonstrated that positivity of the Paneitz-Branson operator is stable under the process of taking connected sums of two closed Riemannian manifolds.

In Section 2 we discuss some preliminary facts about the Paneitz functional. In Section 3 we calculate and verify Theorem 1.1. In Section 4 we prove Theorem 1.2.

\section{Preliminaries}

Recall that the Yamabe constant of any closed manifold of dimension greater than 2 is a finite number and the largest possible Yamabe constant is realized and only realized by the Yamabe constant of the standard round sphere in each dimension. The difficult part is to show that the round sphere is the only one that has the largest Yamabe constant, which was the last step in the resolution of Yamabe problem solved by Schoen in [1984] based on a positive mass theorem of Schoen and Yau. 
We observe that, by (1-3),

$$
\begin{aligned}
\int_{M}(u P[g] u) d v_{g} & =\int_{M} u Q\left[g_{u}\right] u^{\frac{n+4}{n-4}} d v_{g} \\
& =\int_{M} Q\left[g_{u}\right] u^{\frac{2 n}{n-4}} d v_{g}=\int_{M} Q\left[g_{u}\right] d v_{g_{u}},
\end{aligned}
$$

where $g_{u}=u^{\frac{4}{n-4}} g \in[g]$. Hence

$$
\begin{aligned}
\int_{M}(u P[g] & u) d v_{g} \\
& =\int_{M}\left(\left(\frac{(n-4)\left(n^{3}-4 n^{2}+16 n-16\right)}{16(n-1)^{2}(n-2)^{2}} R^{2}-\frac{2(n-4)}{(n-2)^{2}}|\mathrm{Ric}|^{2}\right) d v\right)\left[g_{u}\right] \\
& \leq c_{n} \int_{M}\left(\left(R^{2}\right) d v\right)\left[g_{u}\right],
\end{aligned}
$$

where

$$
c_{n}=\frac{(n-4)\left(n^{3}-4 n^{2}+16 n-1 g\right)}{16(n-1)^{2}(n-2)^{2}}-\frac{2(n-4)}{n(n-2)^{2}} .
$$

When we consider a Yamabe metric $g_{u}$, we have

$$
\frac{\int_{M}(R d v)\left[g_{u}\right]}{\operatorname{vol}\left(M, g_{u}\right)^{\frac{n-2}{n}}}=Y \operatorname{vol}\left(M, g_{u}\right)^{\frac{2}{n}} \leq n(n-1) \operatorname{vol}\left(S^{n}, g_{0}\right)^{\frac{2}{n}},
$$

and since $Y$ and $c_{n}$ are nonnegative by hypothesis, we have

$$
\begin{aligned}
\frac{\int_{M}(u P[g] u) d v_{g}}{\operatorname{vol}\left(M, g_{u}\right)^{\frac{n-4}{n}}} & \leq c_{n} Y^{2} \operatorname{vol}\left(M, g_{u}\right)^{\frac{4}{n}} \leq c_{n}(n(n-1))^{2} \operatorname{vol}\left(S^{n}, g_{0}\right)^{\frac{4}{n}} \\
& =\frac{\int_{S^{n}}(Q d v)\left[g_{0}\right]}{\operatorname{vol}\left(S^{n}, g_{0}\right)^{\frac{n-4}{n}}}=\lambda\left(S^{n},\left[g_{0}\right]\right) .
\end{aligned}
$$

Consequently we obtain:

Lemma 2.1. Let $\left(M^{n}, g\right)$ be a closed Riemannian manifold of dimension greater than 5 with nonnegative Yamabe constant. Then

$$
\lambda\left(M^{n},[g]\right) \leq \lambda\left(S^{n},\left[g_{0}\right]\right)
$$

and the equality holds if and only if $(M, g)$ is conformally equivalent to the standard round sphere $\left(S^{n}, g_{0}\right)$.

On the other hand, by some choices of testing functions similar to the ones used to estimate the Yamabe functional, we get: 
Lemma 2.2. Let $\left(M^{n}, g\right)$ be a closed Riemannian manifold of dimension greater than 4. Then

$$
-\infty<\lambda\left(M^{n},[g]\right) \leq \lambda\left(S^{n},\left[g_{0}\right]\right),
$$

where $g_{0}$ is the standard round metric on the sphere $S^{n}$.

Proof. The Paneitz constant is easily seen to be bounded from below, because, by $(1-2)$,

$$
\begin{aligned}
& \int_{M}(u P[g] u) d v= \\
& \int_{M}|\Delta u|^{2} d v+a_{n} \int_{M} R|\nabla u|^{2} d v-\frac{4}{n-4} \int_{M} \operatorname{Ric}(\nabla u, \nabla u) d v+\int_{M} Q u^{2} d v,
\end{aligned}
$$

where

$$
a_{n}=\frac{(n-2)^{2}+4}{2(n-1)(n-2)} .
$$

It suffices to estimate (2-3) for nonnegative functions such that

$$
\int_{M} u^{\frac{2 n}{n-4}} d v=1
$$

Hence, by Hölder's inequality,

$$
\begin{aligned}
\int_{M}(u P[g] u) d v & \geq \int_{M}|\Delta u|^{2} d v-C_{1} \int_{M}|\nabla u|^{2} d v-C_{2} \int_{M} u^{2} d v \\
& \geq \int_{M}|\Delta u|^{2} d v-C_{1} \int_{M}(-\Delta u) u d v-C_{2} \int_{M} u^{2} d v \\
& \geq \frac{1}{2} \int_{M}|\Delta u|^{2} d v-\frac{1}{2} C_{1}^{2} \int_{M} u^{2} d v-C_{2} \int_{M} u^{2} d v \\
& \geq-\left(\frac{1}{2} C_{1}^{2}+C_{2}\right)\left(\int_{M} u^{\frac{2 n}{n-4}} d v\right)^{\frac{n-4}{n}} \operatorname{vol}(M, g)^{\frac{4}{n}} \\
& \geq-\left(\frac{1}{2} C_{1}^{2}+C_{2}\right) \operatorname{vol}(M, g)^{\frac{4}{n}},
\end{aligned}
$$

for some constants $C_{1}, C_{2}>0$ depending on $\left(M^{n}, g\right)$.

To estimate the upper bound we choose to work in geodesic normal coordinates in a very small geodesic ball $B_{2 \epsilon} \subset M$ and transplant the rescaled round sphere metric. Let $B_{2 \epsilon}(0) \subset R^{n}$ and

$$
g_{i j}(x)=\delta_{i j}+O\left(|x|^{2}\right) \quad \text { for all } x \in B_{2 \epsilon}(0) .
$$


Define a smooth nonnegative function $u_{\epsilon}$ on $M$ by

$$
u_{\epsilon}(x)=\left\{\begin{array}{ll}
\left(\frac{2 \epsilon^{3}}{\epsilon^{6}+|x|^{2}}\right)^{\frac{n-4}{2}} & \text { for } x \in B_{\epsilon}(0), \\
0 & \text { for } x \notin B_{2 \epsilon}(0)
\end{array} .\right.
$$

It is easily calculated that

$$
\begin{aligned}
\int_{M}\left(u_{\epsilon} P[g] u_{\epsilon}\right) d v & =\int_{B_{\epsilon}(0)}\left|\Delta u_{\epsilon}\right|^{2} d x+o(1) \\
& =\int_{R^{n}}\left|\Delta\left(\frac{2 \epsilon^{3}}{\epsilon^{6}+|x|^{2}}\right)^{\frac{n-4}{2}}\right|^{2} d x+o(1) \\
& =\int_{R^{n}}\left|\Delta\left(\frac{2}{1+|x|^{2}}\right)^{\frac{n-4}{2}}\right|^{2} d x+o(1)
\end{aligned}
$$

and

$$
\begin{aligned}
\int_{M} u_{\epsilon}^{\frac{2 n}{n-4}} d v & =\int_{B_{\epsilon}(0)} u_{\epsilon}^{\frac{2 n}{n-4}} d x+o(1) \\
& =\int_{R^{n}}\left(\frac{2 \epsilon^{3}}{\epsilon^{6}+|x|^{2}}\right)^{n} d x+o(1) \\
& =\int_{R^{n}}\left(\frac{2}{1+|x|^{2}}\right)^{n} d x+o(1) .
\end{aligned}
$$

Therefore

$$
\wp\left(u_{\epsilon}\right)=\frac{\int_{M}\left(u_{\epsilon} P[g] u_{\epsilon}\right) d v}{\left(\int_{M} u_{\epsilon}^{\frac{2 n}{n-4}} d v\right)^{\frac{n-4}{n}}}=\frac{\int_{R^{n}}|\Delta s|^{2} d x}{\left(\int_{R^{n}} s^{\frac{2 n}{n-4}} d x\right)^{\frac{n-4}{n}}}+o(1),
$$

where $s=\left(\frac{2}{1+|x|^{2}}\right)^{\frac{n-4}{2}}$. Thus, taking $\epsilon \rightarrow 0$, we arrive at

$$
\lambda(M,[g]) \leq \lambda\left(S^{n},\left[g_{0}\right]\right) .
$$

One interesting question would be whether $(M, g)$ is conformally equivalent to $\left(S^{n}, g_{0}\right)$ when $\lambda(M,[g])=\lambda\left(S^{n},\left[g_{0}\right]\right)$ without assuming the Yamabe constant of $(M, g)$ is nonnegative. In other words one would be interested in searching for some analogue of a positive mass theorem of Schoen and Yau here if it make any sense.

\section{Connected sums and the Paneitz constant}

In this section we will calculate the Paneitz functional on a connected sum of two closed manifolds and verify Theorem 1.1. Let $(M, g)$ be a closed manifold of 
dimension higher than 4. Fix a point $p \in M$ and let

$$
f_{\delta}= \begin{cases}0 & \text { for } x \in B_{\delta}(p), \\ 1 & \text { for } x \in M \backslash B_{2 \delta}(p),\end{cases}
$$

be a family of smooth functions. We may ask that

$$
0 \leq f_{\delta} \leq 1, \quad\left|\nabla f_{\delta}\right|<\frac{C_{0}}{\delta}, \quad\left|\Delta f_{\delta}\right|<\frac{C_{0}}{\delta^{2}}
$$

for some number $C_{0}>0$.

Lemma 3.1. Let $(M, g)$ be a closed manifold of dimension greater than 4. Let $u \in C_{+}{ }^{\infty}(M)$ be given. Then $u_{\delta}=f_{\delta} u \in C_{+}{ }^{\infty}(M)$ and

$$
\wp_{g}\left(u_{\delta}\right)=\wp_{g}(u)+o(1)
$$

as $\delta \rightarrow 0$.

Proof. We simply calculate, for a fixed $\delta>0$, by (2-6) and (3-2),

$$
\begin{aligned}
& \text { (3-4) } \int_{M}\left(u_{\delta} P[g] u_{\delta}\right) d v \\
& =\int_{M}\left|\Delta u_{\delta}\right|^{2} d v+a_{n} \int_{M} R\left|\nabla u_{\delta}\right|^{2} d v-\frac{4}{n-4} \int_{M} \operatorname{Ric}\left(\nabla u_{\delta}, \nabla u_{\delta}\right) d v+\int_{M} Q u_{\delta}^{2} d v \\
& =\int_{M}(u P[g] u) d v+o(1)
\end{aligned}
$$

and

$$
\int_{M} u_{\delta}^{\frac{2 n}{n-4}} d v=\int_{M} u^{\frac{2 n}{n-4}} d v+o(1)
$$

as $\delta \rightarrow 0$.

Now let us consider the connected sum of two closed Riemannian manifolds. Let $\left(M_{1}, g_{1}\right)$ and $\left(M_{2}, g_{2}\right)$ be two compact Riemannian manifolds without boundary of dimension $n \geq 5$. For $x_{1} \in M_{1}$ and $x_{2} \in M_{2}$, let $B_{\delta_{1}}\left(x_{1}\right) \subset M_{1}$ and $B_{\delta_{2}}\left(x_{2}\right) \subset M_{2}$ be geodesic balls respectively. To make the connected sum one simply takes off the open balls $B_{\frac{1}{2} \delta_{1}}\left(x_{1}\right)$ and $B_{\frac{1}{2} \delta_{2}}\left(x_{2}\right)$ from $M_{1}$ and $M_{2}$, identify $\partial B_{\frac{1}{2} \delta_{1}}\left(x_{1}\right)$ with $\partial B_{\frac{1}{2} \delta_{2}}\left(x_{2}\right)$. Hence

$$
M_{1} \# M_{2}=\left[\left(M_{1} \backslash B_{\frac{1}{2} \delta_{1}}\left(x_{1}\right)\right) \cup\left(M_{2} \backslash B_{\frac{1}{2} \delta_{2}}\left(x_{2}\right)\right)\right] /\left\{\partial B_{\frac{1}{2} \delta_{1}}\left(x_{1}\right) \sim \partial B_{\frac{1}{2} \delta_{2}}\left(x_{2}\right)\right\} .
$$

We may construct a metric $g$ on the connected sum $M_{1} \# M_{2}$ such that $g$ agrees with $g_{1}$ on $M_{1} \backslash B_{\delta_{1}}\left(x_{1}\right)$ and $g_{2}$ on $M_{2} \backslash B_{\delta_{2}}\left(x_{2}\right)$. Notice that topologically $M_{1} \# M_{2}$ does not depend on the value of $\delta_{i}$ when they are sufficiently small. Now let us calculate and estimate the Paneitz functional on the connected sum. 
Theorem 3.2. Let $\left(M_{1}, g_{1}\right)$ and $\left(M_{2}, g_{2}\right)$ be two closed Riemannian manifolds of dimension $n \geq 5$. Then for each $\epsilon>0$, there is a conformal structure $[g]$ on $M_{1} \# M_{2}$ such that

$$
\lambda\left(M_{1} \# M_{2},[g]\right)<\min \left\{\lambda\left(M_{1},\left[g_{1}\right]\right), \lambda\left(M_{2},\left[g_{2}\right]\right)\right\}+\epsilon .
$$

Alternatively, we may find a conformal structure $[g]$ on $M_{1} \# M_{2}$ such that

$$
\lambda(M,[g])<\lambda\left(M_{1},\left[g_{1}\right]\right)+\lambda\left(M_{2},\left[g_{2}\right]\right) 2^{-\frac{n-4}{n}}+\epsilon .
$$

Proof. Let us assume that $\lambda\left(M_{1},\left[g_{1}\right]\right) \leq \lambda\left(M_{2},\left[g_{2}\right]\right)$ and $\epsilon>0$ fixed. By the definition of the Paneitz constant, we know that there is a real number $\delta>0$ and a smooth function $u_{\delta} \in C_{+}{ }^{\infty}(M)$ such that $u_{\delta}$ vanishes on a geodesic ball $B_{\delta}\left(x_{1}\right)$ of radius $\delta$ and centered at $x_{1} \in M_{1}$ and such that

$$
\wp g\left(u_{\delta}\right)<\lambda\left(M_{1},\left[g_{1}\right]\right)+\epsilon .
$$

Let $g$ be a metric on $M=M_{1} \# M_{2}$ which agrees with $g_{1}$, when restricted to $M_{1} \backslash B_{\delta}\left(x_{1}\right)$. And define the function $\tilde{u}_{\delta}$ on $M_{1} \# M_{2}$ as follows:

$$
\begin{cases}\tilde{u}_{\delta}=u_{\delta} & \text { on } M_{1} \backslash B_{\delta}\left(x_{1}\right), \\ \tilde{u}_{\delta}=0 & \text { elsewhere. }\end{cases}
$$

We then have

$$
\wp_{g}\left(\tilde{u}_{\delta}\right)=\frac{\int_{M}\left(\Delta \tilde{u}_{\delta}^{2}+a_{n} R\left|\nabla \tilde{u}_{\delta}\right|^{2}-\frac{4}{n-2} \operatorname{Ric}\left(\nabla \tilde{u}_{\delta}, \nabla \tilde{u}_{\delta}\right)+Q \tilde{u}_{\delta}^{2}\right) d v}{\left(\int_{M} \tilde{u}_{\delta}^{\frac{2 n}{n-4}} d v\right)^{\frac{n}{n-4}}} .
$$

Recalling that $u_{\delta}$ vanishes on $B_{\delta}\left(x_{1}\right)$ we see that

$$
\wp g\left(\tilde{u}_{\delta}\right)=\wp g_{1}\left(u_{\delta}\right)<\lambda\left(M_{1},\left[g_{1}\right]\right)+\epsilon .
$$

Consequently,

$$
\lambda(M,[g])<\lambda\left(M_{1},\left[g_{1}\right]\right)+\epsilon=\min \left(\lambda\left(M_{1},\left[g_{1}\right]\right), \lambda\left(M_{2},\left[g_{2}\right]\right)\right)+\epsilon .
$$

We now proceed to prove (3-8). First, Lemma 3.1 can be used to say that for any fixed $\epsilon>0, x_{1} \in M_{1}, x_{2} \in M_{2}$, we can find two positive reals $\delta_{1}, \delta_{2}$ and smooth functions $u_{\delta_{1}}, u_{\delta_{2}}$, where $u_{\delta_{i}} \in C^{\infty}\left(M_{i}\right)$, with the following properties:

$$
\begin{array}{lll}
u_{\delta_{1}}=0 & \text { on } B_{\delta_{1}}\left(x_{1}\right), & \wp g_{1}\left(u_{\delta_{1}}\right)<\lambda\left(M_{1},\left[g_{1}\right]\right)+\epsilon_{1}, \\
u_{\delta_{2}}=0 & \text { on } B_{\delta_{2}}\left(x_{2}\right), & \wp g_{2}\left(u_{\delta_{2}}\right)<\lambda\left(M_{2},\left[g_{2}\right]\right)+\epsilon_{1},
\end{array}
$$

where $\epsilon_{1}=2^{\frac{-n+4}{n}} \epsilon$. Also, notice that we can assume without loss of generality that the $L^{\frac{2 n}{n-4}}(M)$ norms of $u_{\delta_{1}}$ and $u_{\delta_{2}}$ are normalized. Using the same reasoning as in the proof of (3-7), a metric $g$ on $M_{1} \# M_{2}$ can be constructed such that $g$ 
agrees with $g_{i}$ when restricted to $M_{i} \backslash B_{\delta_{i}}\left(x_{i}\right)$. Let us consider now the function $\tilde{u}$ on $M=M_{1} \# M_{2}$ given by

$$
\tilde{u}= \begin{cases}u_{\delta_{1}} & \text { on } M_{1} \backslash B_{\delta_{1}}\left(x_{1}\right), \\ u_{\delta_{2}} & \text { on } M_{2} \backslash B_{\delta_{2}}\left(x_{1}\right), \\ 0 & \text { elsewhere }\end{cases}
$$

then

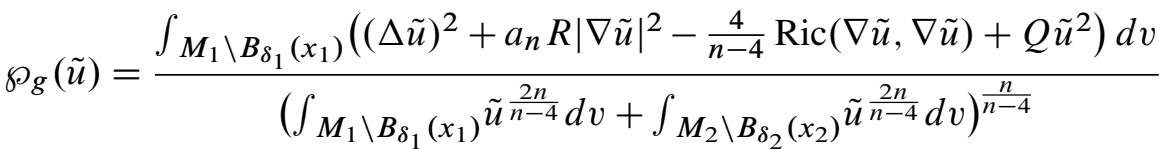

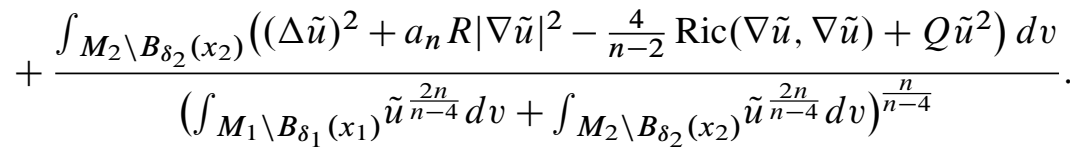

Using (3-9) we then obtain

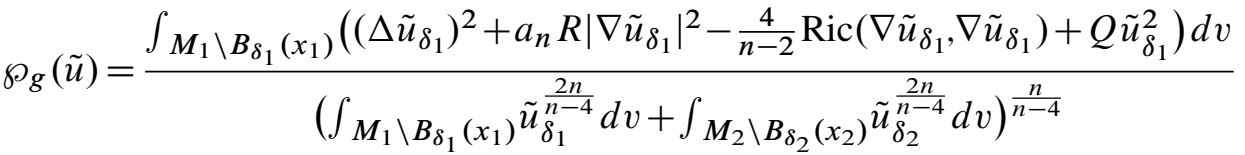

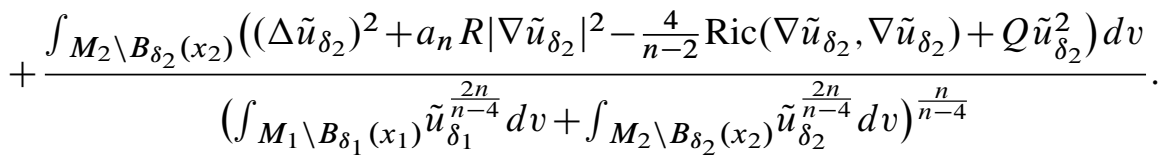

Now, recalling the above stated properties of $u_{\delta_{1}}$ and $u_{\delta_{2}}$, we may also assume

$$
\int_{M_{i} \backslash B_{\delta_{i}}\left(x_{i}\right)} u_{\delta_{i}}^{\frac{2 n}{n-4}} d v=1
$$

and

$$
\begin{aligned}
\wp g_{i}( & \left.u_{\delta_{i}}\right) \\
& =\int_{M_{i} \backslash B_{\delta_{i}}\left(x_{i}\right)}\left(\Delta \tilde{u}_{\delta_{i}}{ }^{2}+a_{n} R\left|\nabla \tilde{u}_{\delta_{i}}\right|^{2}-\frac{4}{n-2} \operatorname{Ric}\left(\nabla \tilde{u}_{\delta_{i}}, \nabla \tilde{u}_{\delta_{i}}\right)+Q \tilde{u}_{\delta_{i}}^{2}\right) d v \\
& <\lambda\left(M_{i},\left[g_{i}\right]\right)+\epsilon_{1} .
\end{aligned}
$$

Thus

$$
\begin{aligned}
\lambda(M,[g]) \leq \wp_{g}(\tilde{u}) & <\left(\lambda\left(M_{1},\left[g_{1}\right]\right)+\lambda\left(M_{2},\left[g_{2}\right]\right)+2 \epsilon_{1}\right) 2^{-\frac{n-4}{n}} \\
& =\left(\lambda\left(M_{1},\left[g_{1}\right]\right)+\lambda\left(M_{2},\left[g_{2}\right]\right)\right) 2^{-\frac{n-4}{n}}+\epsilon .
\end{aligned}
$$

\section{Connected sums and the Paneitz invariants}

Kobayashi in [1987] showed that the Yamabe invariant of connected sum of two manifolds is greater than or equal to the smaller of the Yamabe invariants of the 
two. The aim of this section is to generalize this result of Kobayashi to the case of compact manifolds of dimension $n \geq 5$, and with the Yamabe invariant $Y(M)$ replaced by it's fourth-order analogue the Paneitz invariant $\lambda(M)$. Namely, we have

Theorem 4.1. Let $M_{1}$ and $M_{2}$ be closed manifolds of dimension $n \geq 5$. If $\lambda\left(M_{1}\right)>0$ and $\lambda\left(M_{2}\right)>0$ then

$$
\lambda\left(M_{1} \# M_{2}\right) \geq \min \left\{\lambda\left(M_{1}\right), \lambda\left(M_{2}\right)\right\} .
$$

We will basically follow the approach taken in [Kobayashi 1987]. First we consider the Paneitz invariant on the disjoint union of compact manifolds. Take two $n$-manifolds with conformal structures, say $\left(M_{1},\left[g_{1}\right]\right)$ and $\left(M_{2},\left[g_{2}\right]\right)$. We write $(M,[g])=\left(M_{1},\left[g_{1}\right]\right) \sqcup\left(M_{2},\left[g_{2}\right]\right)$ if $M$ is the disjoint union of $M_{1}$ and $M_{2}$, and $g_{i}=\left\{\left.g\right|_{M_{i}} ; g \in[g]\right\}$ for $i=1,2$. Let $u$ be a smooth nonnegative function on $M$. Since $M$ is the disjoint union of $M_{1}$ and $M_{2}$ it follows that we can write $u=u_{1}+u_{2}$, where $u_{i}=0$ on $M_{j}$, where $i \neq j$ and where $u_{i}$ is a nonnegative smooth function on $M_{i}$. If we assume that $\lambda\left(M_{i},\left[g_{i}\right]\right) \geq 0$ for $i=1,2$, then it can easily be seen that

$$
\lambda(M,[g])=\min \left\{\lambda\left(M_{1},\left[g_{1}\right]\right), \lambda\left(M_{2},\left[g_{2}\right]\right)\right\} .
$$

Due to Lemma 2.2, we can assume that $\lambda\left(M_{1}\right)$ and $\lambda\left(M_{2}\right)$ are finite; and we can use the above equation to conclude that

$$
\lambda(M)=\min \left\{\lambda\left(M_{1}\right), \lambda\left(M_{2}\right)\right\} .
$$

Let $M$ be a compact manifold of dimension $n \geq 5$, and $p_{1}$ and $p_{2}$ two points of $M$. We take off two small balls around $p_{1}$ and $p_{2}$, and then attach a handle instead, the handle being topologically the product of a line segment and $S^{n-1}$. The new manifold obtained in this way will be denoted by $\bar{M}$. Let $M_{1}$ and $M_{2}$ be Riemannian manifolds and let $M_{1} \sqcup M_{2}$ denote the disjoint union of $M_{1}$ and $M_{2}$. If $M=M_{1} \sqcup M_{2}$ and $p_{1}$ and $p_{2}$ are taken from $M_{1}$ and $M_{2}$ respectively, then $\bar{M}=M_{1} \# M_{2}$. Therefore we see that in order to prove Theorem 4.1 it suffices to show

$$
\lambda(\bar{M}) \geq \lambda(M) .
$$

Proof of Theorem 4.1. Let $\epsilon$ be an arbitrary positive number, which will be fixed throughout. First, we take a metric $g$ on $M$ such that

$$
\lambda(M,[g])>\lambda(M)-\epsilon .
$$

Due to continuity considerations we may assume that $[g]$ is conformally flat around the points $p_{1}$ and $p_{2}$. Then there is a function $\gamma \in C^{\infty}\left(M \backslash\left\{p_{1}, p_{2}\right\}\right)$ and $g \in[g]$ such that $\tilde{g}=e^{\gamma} g$ is a complete metric of $M \backslash\left\{p_{1}, p_{2}\right\}$ and that each of the two 
ends is isometric to the half-infinite cylinder $[0, \infty) \times S^{n-1}(1)$. For convenience, we write

$$
\left(M \backslash\left\{p_{1}, p_{2}\right\}, \tilde{g}\right)=[0, \infty) \times S^{n-1}(1) \cup(\tilde{M}, \tilde{g}) \cup[0, \infty) \times S^{n-1}(1),
$$

where $\tilde{M}$ is the complement of the two cylinders. We can glue $(\tilde{M}, \tilde{g})$ and $[0, l] \times$ $S^{n-1}(1)$, along their boundaries to get a smooth Riemannian manifold $\left(\bar{M}, g_{l}\right)$, where $\bar{M}$ is as mentioned in the beginning of the section:

$$
\left(\bar{M}, \bar{g}_{l}\right)=(\tilde{M}, \tilde{g}) \cup[0, l] \times S^{n-1}(1) .
$$

We then have

$$
\lambda\left(\bar{M},\left[g_{l}\right]\right)=\inf _{f>0} \frac{\int_{\bar{M}}\left((\Delta f)^{2}+a_{n} R|\nabla f|^{2}-\frac{4}{n-2} \operatorname{Ric}(\nabla f, \nabla f)+Q f^{2}\right) d v}{\left(\int_{\bar{M}} f^{\frac{2 n}{n-4}} d v\right)^{\frac{n}{n-4}}},
$$

So, take a positive function $f_{l} \in C^{\infty}(\bar{M})$ such that

$$
\begin{aligned}
\int_{\bar{M}}\left(\left(\Delta f_{l}\right)^{2}+a_{n} R\left|\nabla f_{l}\right|^{2}-\frac{4}{n-2} \operatorname{Ric}\left(\nabla f_{l}, \nabla f_{l}\right)\right. & \left.+Q f^{2}\right) d v \\
& <\lambda\left(\bar{M},\left[g_{l}\right]\right)+\frac{1}{l+1}
\end{aligned}
$$

and

$$
\int_{\bar{M}} f_{l}^{\frac{2 n}{n-4}} d v=1
$$

Lemma 4.2. There is a section, say $\left\{t_{l}\right\} \times S^{n-1}$, in the cylindrical part of $\bar{M}$ such that

$$
\int_{\left\{t_{l}\right\} \times S^{n-1}}\left(\left(\Delta f_{l}\right)^{2}+a_{n} R\left|\nabla f_{l}\right|^{2}-\frac{4}{n-2} \operatorname{Ric}\left(\nabla f_{l}, \nabla f_{l}\right)+Q f^{2}\right) d v<\frac{B}{l},
$$

where $B$ is a constant independent of $l$.

Proof. Using (4-4) we have

$$
\begin{aligned}
& \int_{S^{n-1} \times[0, l]}\left(\left(\Delta f_{l}\right)^{2}+a_{n} R\left|\nabla f_{l}\right|^{2}-\frac{4}{n-2} \operatorname{Ric}\left(\nabla f_{l}, \nabla f_{l}\right)+Q f_{l}^{2}\right) d v \\
& <\lambda\left(\bar{M},\left[g_{l}\right]\right)+\frac{1}{1+l} \\
& \quad-\int_{\tilde{M}}\left(\left(\Delta f_{l}\right)^{2}+a_{n} R\left|\nabla f_{l}\right|^{2}-\frac{4}{n-2} \operatorname{Ric}\left(\nabla f_{l}, \nabla f_{l}\right)+Q f_{l}^{2}\right) d v .
\end{aligned}
$$

Now suppose that $\int_{\bar{M}}\left|\nabla f_{l}\right|^{2} d v$ goes to infinity as $l \rightarrow \infty$. It would follow that $\int_{\bar{M}}\left(\Delta f_{l}\right)^{2} \rightarrow \infty$ as $l \rightarrow \infty$ and that this divergence is much faster than the divergence of $\int_{\bar{M}}\left|\nabla f_{l}\right|^{2} d v$. But this implies that $\int_{\bar{M}} f_{l} P_{l} f_{l} d v>\lambda\left(\bar{M},\left[g_{l}\right]\right)+\frac{1}{l+1}$ for large $l$, which forces a contradiction (here $P_{l}$ is the Paneitz-Branson operator 
of the metric $g_{l}$.) It follows that there exists a constant $D$ independent of $l$ such that

$$
\int_{\bar{M}} a_{n} R\left|\nabla f_{l}\right|^{2}-\frac{4}{n-2} \operatorname{Ric}\left(\nabla f_{l}, \nabla f_{l}\right) d v \leq D .
$$

Note as well that there exists a constant $E$ such that $-\int_{\bar{M}} Q f_{l}^{2} d v \leq E$. Putting this together we conclude that there exists a $t_{1} \in[0, l]$ such that

$$
\begin{aligned}
l \int_{t_{1} \times S^{n-1}}\left(\left(\Delta f_{l}\right)^{2}+a_{n} R\left|\nabla f_{l}\right|^{2}-\frac{4}{n-2} \operatorname{Ric}\left(\nabla f_{l}, \nabla f_{l}\right)+Q f_{l}^{2}\right) d v & \\
& <\lambda\left(\bar{M},\left[g_{l}\right]\right)+\frac{1}{1+l}+D+E .
\end{aligned}
$$

The lemma follows.

Now we cut off $\bar{M}$ on the section $\left\{t_{1} \times S^{n-1}\right\}$, and attach two half-infinite cylinders to it, so $\left(M, \backslash\left\{p_{1}, p_{2}\right\}, \bar{g}\right)$ reappears. But this time we describe it as follows:

$$
\left(M, \backslash\left\{p_{1}, p_{2}\right\}, \bar{g}\right)=[0, \infty) \times S^{n-1}(1) \cup\left(\bar{M}-\left\{t_{1}\right\} \times S^{n-1}, g_{l}\right) \cup[0, \infty) \times S^{n-1}(1) .
$$

We think of the function $f_{l}$ as defined on $\bar{M}-\left\{\left\{t_{l}\right\} \times S^{n-1}\right\}$, and extend it to the whole space $M-\left\{p_{1}, p_{2}\right\}$ as follows: Let $F_{l}$ be $W^{2, \infty}$ function of $\bar{M}-\left\{p_{1}, p_{2}\right\}$ such that

$$
F_{l}=f_{l} \quad \text { on } \bar{M}-\left\{t_{l}\right\} \times S^{n-1}
$$

and

$$
F_{l}(t, x)= \begin{cases}g(t) \tilde{f}_{l}(x) & \text { for }(t, x) \in[0,1] \times S^{n-1}, \\ 0 & \text { for }(t, x) \in[1, \infty] \times S^{n-1},\end{cases}
$$

where $\tilde{f}_{l}=\left.f_{l}\right|_{\left\{t_{l}\right\} \times S^{n-1}} \in C^{\infty}\left(S^{n-1}\right)$ and where $g$ is a smooth function on $[0,1]$ that goes from a value of 1 to a value of 0 , and whose derivative vanishes at 1 . Now it easy to see from (4-4) and the above lemma that

$$
\begin{aligned}
\int_{M \backslash\left\{p_{1}, p_{2}\right\}}\left(\left(\Delta F_{l}\right)^{2}+a_{n} R\left|\nabla F_{l}\right|^{2}-\frac{4}{n-2} \operatorname{Ric}\left(\nabla F_{l}, \nabla F_{l}\right)+\right. & \left.Q F^{2}\right) d v \\
& <\lambda\left(\bar{M},\left[g_{l}\right]\right)+\frac{B}{l},
\end{aligned}
$$

where $B$ is a constant independent of $l$. Obviously from (4-5) we get

$$
\int_{\bar{M} \backslash\left\{p_{1}, p_{2}\right\}} F_{l}^{\frac{2 n}{n-4}} d v>1 .
$$

Therefore, we have

$$
\inf \frac{\int_{M \backslash\left\{p_{1}, p_{2}\right\}}\left((\Delta F)^{2}+a_{n} R|\nabla F|^{2}-\frac{4}{n-2} \operatorname{Ric}(\nabla F, \nabla F)+Q F^{2}\right) d v}{\left(\int_{M \backslash\left\{p_{1}, p_{2}\right\}} F^{\frac{2 n}{n-4}} d v\right)^{\frac{n}{n-4}}} \leq \lambda(\bar{M}),
$$


where the infimum is taken over all nonnegative $W^{2, \infty}$ functions $F$ with compact support. It follows from the choice of the metric $\tilde{g}$ that the left side of the preceding equation is equal to $\lambda(M,[g])$. Since $\epsilon$ can be chosen arbitrarily in (4-2), we conclude $\lambda(M) \leq \lambda(\bar{M})$, which completes the proof.

\section{References}

[Aubin 1976] T. Aubin, "The scalar curvature", pp. 5-18 in Differential geometry and relativity, edited by M. Cahen and M. Flato, Mathematical Phys. and Appl. Math. 3, Reidel, Dordrecht, 1976. MR 55 \#6476 Zbl 0345.53029

[Branson 1987] T. P. Branson, "Group representations arising from Lorentz conformal geometry", $J$. Funct. Anal. 74:2 (1987), 199-291. MR 90b:22016 Zbl 0643.58036

[Chang and Yang 2002] S.-Y. A. Chang and P. C. Yang, "Non-linear partial differential equations in conformal geometry", pp. 189-207 in Proceedings of the International Congress of Mathematicians, I (Beijing, 2002), edited by T. Li, Higher Ed. Press, Beijing, 2002. MR 2004d:53031

[Chang et al. 2004] S.-Y. A. Chang, F. Hang, and P. C. Yang, "On a class of locally conformally flat manifolds”, Int. Math. Res. Not. 2004:4 (2004), 185-209. MR 2005d:53051 Zbl 1137.53327

[Djadli et al. 2000] Z. Djadli, E. Hebey, and M. Ledoux, "Paneitz-type operators and applications", Duke Math. J. 104:1 (2000), 129-169. MR 2002f:58061 Zbl 0998.58009

[Gil-Medrano 1986] O. Gil-Medrano, "Connected sums and the infimum of the Yamabe functional", pp. 160-167 in Differential geometry (Peñíscola, 1985), edited by A. M. Naveira et al., Lecture Notes in Math. 1209, Springer, Berlin, 1986. MR 88a:58206 Zbl 0604.58023

[González 2005] M. d. M. González, "Singular sets of a class of locally conformally flat manifolds", Duke Math. J. 129:3 (2005), 551-572. MR 2006d:53034 Zbl 1088.53023

[Gromov and Lawson 1980] M. Gromov and H. B. Lawson, Jr., "The classification of simply connected manifolds of positive scalar curvature", Ann. of Math. (2) 111:3 (1980), 423-434. MR 81h:53036 Zbl 0463.53025

[Kobayashi 1987] O. Kobayashi, "Scalar curvature of a metric with unit volume", Math. Ann. 279:2 (1987), 253-265. MR 89a:53048 Zbl 0611.53037

[Paneitz 1983] S. M. Paneitz, "A quartic conformally covariant differential operator for arbitrary pseudo-Riemannian manifolds (summary)", preprint, 1983. arXiv 0803.4331

[Schoen 1984] R. Schoen, "Conformal deformation of a Riemannian metric to constant scalar curvature", J. Differential Geom. 20:2 (1984), 479-495. MR 86i:58137 Zbl 0576.53028

[Schoen and Yau 1979] R. Schoen and S. T. Yau, "On the structure of manifolds with positive scalar curvature”, Manuscripta Math. 28:1-3 (1979), 159-183. MR 80k:53064 Zbl 0423.53032

[Trudinger 1968] N. S. Trudinger, "Remarks concerning the conformal deformation of Riemannian structures on compact manifolds", Ann. Scuola Norm. Sup. Pisa (3) 22 (1968), 265-274. MR 39 \#2093 Zbl 0159.23801

[Xu and Yang 2001] X. Xu and P. C. Yang, "Positivity of Paneitz operators", Discrete Contin. Dynam. Systems 7:2 (2001), 329-342. MR 2002d:58043 Zbl 1032.58018

[Yamabe 1960] H. Yamabe, "On a deformation of Riemannian structures on compact manifolds", Osaka Math. J. 12 (1960), 21-37. MR 23 \#A2847 Zbl 0096.37201

Received September 1, 2010. Revised September 17, 2012. 
DAVID RASKE

UNIVERSITY OF MICHIGAN

ANN ARBOR, MI 48104

UNITED STATES

david.t.raske@gmail.com 


\title{
PACIFIC JOURNAL OF MATHEMATICS
}

\author{
msp.org/pjm
}

Founded in 1951 by E. F. Beckenbach (1906-1982) and F. Wolf (1904-1989)

\section{EDITORS}

V. S. Varadarajan (Managing Editor)

Department of Mathematics

University of California

Los Angeles, CA 90095-1555

pacific@math.ucla.edu

Paul Balmer

Department of Mathematics

University of California

Los Angeles, CA 90095-1555

balmer@math.ucla.edu

Daryl Cooper

Department of Mathematics

University of California

Santa Barbara, CA 93106-3080 cooper@math.ucsb.edu

Jiang-Hua $\mathrm{Lu}$

Department of Mathematics

The University of Hong Kong

Pokfulam Rd., Hong Kong jhlu@maths.hku.hk
Don Blasius

Department of Mathematics University of California

Los Angeles, CA 90095-1555

blasius@math.ucla.edu

Robert Finn

Department of Mathematics Stanford University

Stanford, CA 94305-2125

finn@math.stanford.edu

Sorin Popa

Department of Mathematics

University of California

Los Angeles, CA 90095-1555

popa@math.ucla.edu

Paul Yang

Department of Mathematics

Princeton University

Princeton NJ 08544-1000

yang@math.princeton.edu

\section{PRODUCTION}

Silvio Levy, Scientific Editor, production@msp.org

\section{SUPPORTING INSTITUTIONS}

ACADEMIA SINICA, TAIPEI

CALIFORNIA INST. OF TECHNOLOGY

INST. DE MATEMÁTICA PURA E APLICADA

KEIO UNIVERSITY

MATH. SCIENCES RESEARCH INSTITUTE

NEW MEXICO STATE UNIV.

OREGON STATE UNIV.

\author{
STANFORD UNIVERSITY \\ UNIV. OF BRITISH COLUMBIA \\ UNIV. OF CALIFORNIA, BERKELEY \\ UNIV. OF CALIFORNIA, DAVIS \\ UNIV. OF CALIFORNIA, LOS ANGELES \\ UNIV. OF CALIFORNIA, RIVERSIDE \\ UNIV. OF CALIFORNIA, SAN DIEGO \\ UNIV. OF CALIF., SANTA BARBARA
}

\author{
Vyjayanthi Chari \\ Department of Mathematics \\ University of California \\ Riverside, CA 92521-0135 \\ chari@math.ucr.edu \\ Kefeng Liu \\ Department of Mathematics \\ University of California \\ Los Angeles, CA 90095-1555 \\ liu@math.ucla.edu \\ Jie Qing \\ Department of Mathematics \\ University of California \\ Santa Cruz, CA 95064 \\ qing@cats.ucsc.edu
}

These supporting institutions contribute to the cost of publication of this Journal, but they are not owners or publishers and have no responsibility for its contents or policies.

See inside back cover or msp.org/pjm for submission instructions.

The subscription price for 2013 is US \$400/year for the electronic version, and \$485/year for print and electronic.

Subscriptions, requests for back issues and changes of subscribers address should be sent to Pacific Journal of Mathematics, P.O. Box 4163, Berkeley, CA 94704-0163, U.S.A. The Pacific Journal of Mathematics is indexed by Mathematical Reviews, Zentralblatt MATH, PASCAL CNRS Index, Referativnyi Zhurnal, Current Mathematical Publications and the Science Citation Index.

The Pacific Journal of Mathematics (ISSN 0030-8730) at the University of California, c/o Department of Mathematics, 798 Evans Hall \#3840, Berkeley, CA 94720-3840, is published monthly except July and August. Periodical rate postage paid at Berkeley, CA 94704, and additional mailing offices. POSTMASTER: send address changes to Pacific Journal of Mathematics, P.O. Box 4163, Berkeley, CA 94704-0163.

PJM peer review and production are managed by EditFLOW ${ }^{\circledR}$ from Mathematical Sciences Publishers.

PUBLISHED BY

mathematical sciences publishers

nonprofit scientific publishing

http://msp.org/

(C) 2013 Mathematical Sciences Publishers 


\section{PACIFIC JOURNAL OF MATHEMATICS}

Volume $261 \quad$ No. $2 \quad$ February 2013

Geography of simply connected nonspin symplectic 4-manifolds with positive signature

Anar Akhmedov, Mark C. Hughes and B. Doug Park

Schur-Horn theorems in $\mathrm{II}_{\infty}$-factors

283

MARTÍN ARGERAMI and PEDRo MASSEY

Classification of positive solutions for an elliptic system with a higher-order

fractional Laplacian

JiNGBo DOU and CHANGZHENG QU

Bound states of asymptotically linear Schrödinger equations with compactly supported potentials

MingWEN FEI and HUICHENG YiN

Type I almost homogeneous manifolds of cohomogeneity one, III

DANIEL GUAN

The subrepresentation theorem for automorphic representations

MARCELA HANZER

Variational characterizations of the total scalar curvature and eigenvalues of the

Laplacian

Seungsu Hwang, JeOngwook Chang and GabJin Yun

Fill-ins of nonnegative scalar curvature, static metrics, and quasi-local mass

JEFFREY L. JAUREGUI

Operator algebras and conjugacy problem for the pseudo-Anosov automorphisms of a surface

\section{IGOR NIKOLAEV}

Connected sums of closed Riemannian manifolds and fourth-order conformal invariants

DAVID RASKE

Ruled minimal surfaces in the three-dimensional Heisenberg group

Heayong Shin, Young Wook Kim, Sung-Eun Koh, Hyung Yong LeE and SEONG-DEOG YANG

$G$-bundles over elliptic curves for non-simply laced Lie groups and configurations of lines in rational surfaces

MANG XU and JIAJIN ZHANG 